\title{
Análise de óbitos domiciliares e hospitalares por causas respiratórias e cardiovasculares durante a pandemia da COVID-19 em Minas Gerais
}

\author{
Analysis of home and hospital deaths from respiratory and \\ cardiovascular causes during the COVID-19 pandemic in Minas Gerais
}

\author{
Thiago Henrique Evangelista \\ Alves (iD) \\ Tafarel Andrade de Souza (iD \\ Samyla de Almeida Silva (iD) \\ Nayani Alves Ramos \\ Stefan Vilges de Oliveira* (D)
}

Universidade Federal de Uberlândia (UFU), Uberlândia, MG, Brasil

\section{* E-mail: stefanbio@yahoo.com.br}

Recebido: 24 jul 2020

Aprovado: 18 ago 2020

\section{RESUMO}

Introdução: A pandemia pela COVID-19 em Minas Gerais acarretou a diminuição da procura por atendimentos médicos, como consultas ambulatoriais, exames e cirurgias eletivas, como uma das estratégias de contingência. Objetivo: Analisar as mortes por causas respiratórias e cardiovasculares durante o primeiro semestre de 2020 ocorridas em domicílios e em hospitais de Minas Gerais. Método: As causas de óbitos foram analisadas por meio dos dados do Portal da Transparência do Registro Civil nas semanas epidemiológicas 1 a 23. Resultados: Constatou-se que, comparativamente ao mesmo período de 2019, houve acréscimo nas mortes domiciliares por pneumonia $(17,59 \%)$, insuficiência respiratória $(20,65 \%)$, síndrome respiratória aguda grave $(20,69 \%)$, sepse $(31,91 \%)$, infarto agudo do miocárdio $(15,56 \%)$, acidente vascular cerebral $(18,29 \%)$ e por outras causas cardiovasculares $(18,44 \%)$ em 2020 . Já em relação aos óbitos hospitalares, constatou-se redução das mortes por pneumonia $(6,08 \%)$, por insuficiência respiratória $(3,59 \%)$ e por sepse $(2,21 \%)$, apesar do incremento de $338,30 \%$ por síndrome respiratória aguda grave. Além disso, encontrou-se decréscimo de 3,02\% dos óbitos por acidente vascular cerebral e crescimento de 3,41\% por infarto agudo do miocárdio e de 2,93\% para outras causas cardiovasculares. Em relação à faixa etária e ao sexo, independentemente do local do óbito, predominaram homens com idade entre 75 e 80 anos. Conclusões: Acredita-se que parte desses óbitos esteja relacionada com a reestruturação da dinâmica de atendimento hospitalar associado ao medo de procurar assistência e se infectar pelo novo coronavírus. Conclui-se que houve aumento de mortes domiciliares e redução das mortes hospitalares em 2020 por causas respiratórias e cardiovasculares em meio ao contexto da pandemia.

PALAVRAS-CHAVE: Pandemia; Coronavírus; Mortes; Doenças Pulmonares; Doenças Cardíacas; Minas Gerais

\section{ABSTRACT}

Introduction: The COVID-19 pandemic in Minas Gerais (MG) resulted in a decrease of the demand for healthcare, such as outpatient consultations, exams and elective surgeries, as one of the contingency strategies. Objective: To analyze deaths from respiratory and cardiac causes during the first half of 2020 occurring in households and hospitals in MG. Method: The causes of deaths were analyzed using data from the Civil Registry Transparency Portal (ARPEN) in epidemiological weeks $1^{\text {st }}$ to $23^{\text {th }}$. Results: It was found that, compared to the same period in 2019, there was an increase in home deaths from pneumonia $(17.59 \%)$, respiratory failure $(20.65 \%)$, severe acute respiratory syndrome (20.69\%), sepsis (31.91\%), acute myocardial infarction (15.56\%), stroke (18.29\%) and other cardiovascular causes $(18.44 \%)$ in 2020 . In relation to hospital deaths, a reduction in deaths from pneumonia $(6.08 \%)$, from respiratory failure $(3.59 \%)$ and from sepsis $(2.21 \%)$ was observed, despite an increase of $338.30 \%$ for SARS. In addition, there was a $3.02 \%$ 
decrease in deaths from strokes, and a 3.41\% increase in AMI and $2.93 \%$ for other cardiovascular causes. In relation to age and gender, regardless of the place of death, men aged between 75 and 80 years predominated. Conclusions: It is believed that part of these deaths are related to the restructuring of the dynamics of hospital care, associated with the fear of seeking assistance and becoming infected by the new coronavirus. In conclusion that there was an increase in home deaths and a reduction in hospital deaths in 2020 due to respiratory and cardiac causes in the context of the pandemic.

KEYWORDS: Pandemic; Coronavirus; Deaths; Lung Diseases; Heart Diseases; Minas Gerais

\section{INTRODUÇÃO}

O novo coronavírus surgiu em dezembro de 2019 na cidade de Wuhan, província de Hubei, na China. Inicialmente, foi caracterizada como uma pneumonia consequente de síndrome respiratória aguda pelo vírus SARS-CoV-2. Em fevereiro de 2020, a Organização Mundial da Saúde (OMS) oficializou o nome como doença do coronavírus 2019 (COVID-19) ${ }^{1}$.

Até o fim do primeiro semestre de 2020, já foram confirmados 8.317.055 de acometidos em todo o mundo, com 447.581 óbitos. Os Estados Unidos da América (EUA) ocupam o primeiro lugar em número de casos, com 2.159.446 de ocorrências dessa doença. Nesse mesmo período, o Brasil ocupou a segunda posição desse ranking com 955.377 acometidos $^{2}$.

A região Sudeste do Brasil é a que apresenta maior número de casos (324.604; 35,16\%), atrás apenas do Nordeste. 0 estado de Minas Gerais (MG), localizado nessa área, é o décimo quarto no ranking do país $(22.024 ; 2,38 \%)$. Porém, as cinco últimas projeções desse estado, realizadas em 11 de maio, 18 de maio, 27 de maio, 4 de junho e 10 de junho se sobrepõem, isto é, não se observa diminuição da velocidade de progressão da pandemia ${ }^{3}$. No final de abril, MG contabilizava apenas 1.021 casos, o que demonstra um aumento exponencial de 21.003 acometidos apenas em dois meses ${ }^{3}$.

Outra preocupação em relação ao estado de MG deve-se às crescentes manifestações de síndrome respiratória aguda grave (SRAG). Segundo dados do InfoGripe ${ }^{4}$, a incidência de SRAG apresenta alto índice ao se considerar o histórico do estado. Esse excesso de óbitos no estado é na ordem de quase $650 \%$, o que denota uma ocorrência expressiva dessa síndrome, e com um crescimento concomitante à manifestação de COVID-19. Pela semelhança entre sinais e sintomas entre COVID-19 e SRAG, acredita-se que esse aumento se dê, na verdade, por subnotificação de óbitos pelo novo coronavírus ${ }^{5}$.

O primeiro caso com diagnóstico clínico para o novo coronavírus no Sistema Único de Saúde (SUS) de MG ocorreu na segunda semana de março. A partir dessa data, há uma média de quase 120 internações/dia em leitos clínicos e de, aproximadamente, 27 internações/dia em leitos de unidade de terapia intensiva (UTI). Nesse cenário, o tempo médio de permanência em leitos de terapia intensiva é de 14,2 dias e de 8,77 dias em leitos clíni$\cos ^{3}$. O Ministério da Saúde (MS) do Brasil tem como política de manejo das pessoas com COVID-19 o isolamento em domicílio, visto que o paciente não requer acomodação hospitalar se apresentar as formas leve e moderada da doença ${ }^{6}$.

Nesse contexto, devido às orientações de manter-se o distanciamento social, a Secretaria de Estado de Saúde (SES) de MG estimulou e desenvolveu plataformas que permitissem o atendimento online baseado na telemedicina. Apesar de o foco ser o novo coronavírus, pessoas com diversos sinais e sintomas podem passar por esse processo ${ }^{7}$. Além disso, como estratégia de contenção da pandemia, muitos serviços hospitalares não urgentes foram suspensos, como consultas, exames ou cirurgias eletivas. Isso evita que pessoas que não estejam acometidas pela COVID-19 frequentem unidades de saúde e corram o risco de se infectarem, além de liberar leitos para os pacientes que já estão com o diagnóstico dessa doença ${ }^{8}$. Dessa forma, indivíduos com afecções que exijam acompanhamento contínuo, como as com causas similares às da COVID-19 e as com doenças cardiovasculares, podem ter sido afetados com essa medida, o que resulta em variação no perfil epidemiológico das mortes em relação aos seus locais.

Nesse sentido, o presente artigo teve como objetivo analisar as causas dos óbitos e suas ocorrências domiciliares ou hospitalares no estado de MG em meio ao contexto da pandemia pela COVID-19.

\section{MÉTODO}

Trata-se de um estudo descritivo e quantitativo dos registros cartoriais dos óbitos domiciliares e hospitalares registrados no estado de MG no Brasil. Essa unidade da federação tem uma população estimada, segundo o Instituto Brasileiro de Geografia e Estatística (IBGE), de 21.168.791 pessoas e um território de $586.521,121 \mathrm{~km}^{2}$ no ano de 2019. Seu Índice de Desenvolvimento Humano (IDH) é de 0,731 , e a população de 0 a 15 anos representa $22,25 \%$, a de 15 a 64 anos, $69,31 \%$ e o grupo acima dos 65 anos de idade, $8,12 \%, 10$.

Os dados foram coletados no Portal da Transparência Civil em 6 de julho de 2020. Esse é um site de livre acesso, que foi desenvolvido para disponibilizar ao cidadão dados e informações estatísticas sobre nascimentos, casamentos e óbitos, formando uma Central de Informações do Registro Civil (CRC) no país ${ }^{11}$. As estatísticas apresentadas nesse portal são oriundas das Declarações de Óbito (DO), inclusive as relacionadas à COVID-19, registradas nos Cartórios, as quais apresentam apenas uma causa para cada óbito. Devido à pandemia pela COVID-19, foram criadas 
nesse portal duas seções específicas para se averiguar com mais detalhes os óbitos por essa doença, denominadas de "Especial COVID-19" e de "Painel COVID Registral”12,13.

Foram selecionadas as mortes por causas respiratórias e causas cardiovasculares do período de 1 de janeiro a 6 de junho dos anos de 2019 e 2020. O estado escolhido para a análise foi MG, e foram discriminados os diferentes locais de óbitos: hospitais e domicílios. Para avaliar o padrão total dessas mortes anterior e posterior ao período de aumento de mortes pela COVID-19, a análise foi dividida em dois recortes: o das semanas epidemiológicas (SE) 1 a 11 e outro que considerou as SE 12 a 23. Foram coletadas também, em 14 de julho de 2020, informações sobre a faixa etária e o sexo por causa de óbito selecionada. As estatísticas aqui apresentadas baseiam-se nas DO relacionadas à COVID-19 registradas nos cartórios, as quais apresentam apenas uma causa para cada óbito ${ }^{12}$.

Na DO, a causa mencionada na última linha será considerada como a razão básica da morte, de acordo com as regras da CID10 , quando a sequência de causas informada pelo médico tiver sido preenchida corretamente. São registrados também outros motivos ou condições significativas que podem ter contribuído para o óbito. Nas DO avaliadas neste artigo, selecionou-se aquelas que continham motivo da morte suspeita ou confirmada por COVID-19, bem como outras causas relacionadas a essa doença como: SRAG, pneumonia, insuficiência respiratória (IR), septicemia (sepse/choque séptico). Foram selecionadas também causas cardiovasculares como: acidente vascular cerebral (AVC), infarto agudo do miocárdio (IAM) e causas cardiovasculares inespecíficas (morte súbita ou parada cardiorrespiratória, associada com hipertensão arterial, bloqueio atrioventricular, arritmia cardíaca, taquicardia supraventricular, entre outros). Quanto ao "Local de Óbito", os dados relacionados ao local de falecimento constante nas DO foram atestados pelo médico que a preencheu ${ }^{13,14,15}$.

Para análise estatística dos dados coletados, utilizou-se o teste t-Student para amostras independentes, com o propósito de comparar as médias dos óbitos por causas respiratórias em domicílio dos anos 2019 e 2020 nas SE de 1 a 23, e também para comparar as médias dos óbitos por causas cardiovasculares em domicílio dos anos 2019 e 2020, utilizando o software GraphPad Prism 7 (GraphPad Software, Inc. San Diego, CA). Valor de $p<0,05$ foi considerado estatisticamente significativo. Todos os gráficos foram elaborados utilizando o software GraphPad Prism 7. 0 excesso de mortes refere-se ao número total de óbitos no período das SE 1 a 23 em 2020 subtraído do número total de óbitos no mesmo período em 2019 para cada causa analisada.

Os dados coletados foram analisados em planilha eletrônica por estatística descritiva e apresentados em números brutos e frequência relativa. Por tratar-se de dados secundários de acesso público, a submissão ao Comitê de Ética em Pesquisa foi dispensada.

\section{RESULTADOS}

Ao se analisar as mortes por causas respiratórias em domicílio, verificou-se aumento significativo da média em $2020(79,83 \pm 4,151)$ em comparação com a média de óbitos em 2019 (66,57 $\pm 2,349)$ $(p<0,0080)$. E, quando se avaliou os óbitos por causas cardiovasculares em domicílio, encontrou-se acréscimo significativo da média em $2020(93,96 \pm 4,346)$ em comparação com a média de óbitos em $2019(80,04 \pm 2,792)(p<0,0100)$.

Ao se avaliar as doenças respiratórias em domicílio das SE 1 a 11 de 2020, em contraposição ao ano de 2019, verificou-se o aumento de 81 mortes $(17,02 \%)$, e, durante as SE 12 a 23 , o acréscimo de 206 mortes (32,54\%); quando analisado o cenário hospitalar das SE 1 a 11, encontrou-se aumento de 684 mortes $(8,11 \%)$ e, durante as SE 12 a 23, redução de 1.295 óbitos $(-12,42 \%)$ (Figura 1A). Já para as causas cardiovasculares em domicílio das SE 1 a 11 de 2020, houve aumento de 22 óbitos $(2,67 \%)$, durante as SE 12 a 23, ocorreu acréscimo de 298 mortes $(29,27 \%)$ e, quando se observou as doenças cardiovasculares em ambientes hospitalares das SE 1 a 11, foi identificado aumento de 179 mortes $(6,43 \%)$, e, durante as SE 12 a 23, redução de 111 óbitos (-3,31\%) (Figura 1B).

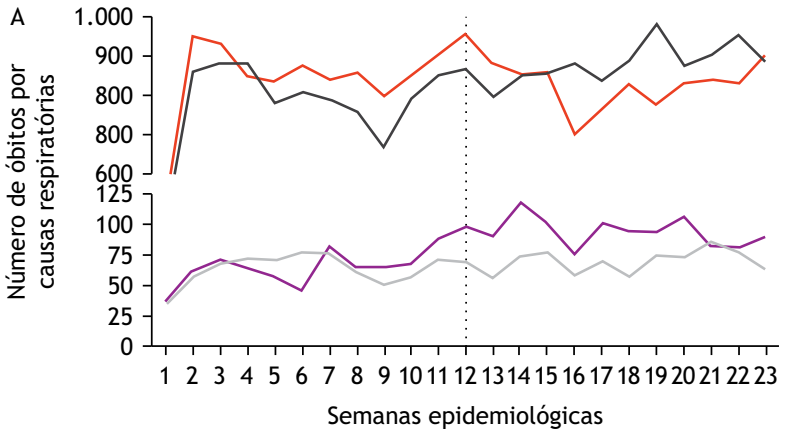

- Mortes em domicílios - 2019 - Mortes em hospitais - 2019 - Mortes em domicílios - 2020 - Mortes em hospitais - 2020

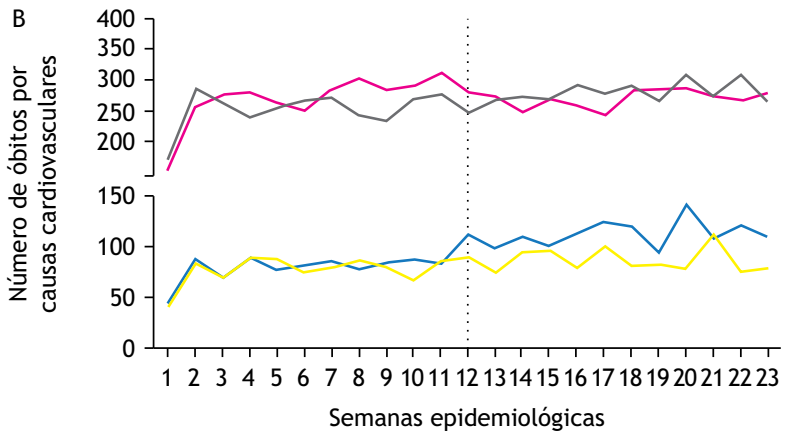

Mortes em domicílios - 2019 - Mortes em hospitais - 2019 - Mortes em domicílios - 2020 - Mortes em hospitais - 2020

Figura 1. Distribuição de óbitos por causas respiratórias e cardiovasculares em hospitais e em domicílios de acordo com o Portal da Transparência do Registro Civil (ARPEN). (A) Óbitos por causas respiratórias em hospitais e em domicílios nos anos de 2019 e 2020, durante as semanas epidemiológicas (SE) de 1 a 23 no estado de Minas Gerais. (B) Óbitos por causas cardiovasculares em hospitais e em domicílios nos anos de 2019 e 2020 , durante as SE de 1 a 23 no estado de Minas Gerais. Linha preta tracejada na SE 12 indica o marco do aumento de óbitos pela COVID-19 no estado de Minas Gerais, Brasil. 
A discriminação das mortes domiciliares por causas respiratórias em 2020 com relação a 2019 revelou aumento de 17,59\% por pneumonia (Figura 2A), com uma maior predominância (50,42\%) entre mulheres de 90 a 99 anos de idade (Fig. 2B). Também se identificou acréscimo de $20,65 \%$ dos óbitos por IR (Figura 2C), predominante em homens $(50,49 \%)$ com idades entre 80 e 89 anos (Figura 2D). Notou-se o incremento de $31,91 \%$ de mortes por sepse (Figura 2E), mas essas tiveram maior ocorrência em mulheres (55,91\%) entre 80 e 89 anos de idade (Figura 2F). Em relação à SRAG, encontrou-se adição de 20,69\% (Figura $2 \mathrm{G}$ ), com maior preponderância em homens $(65,71 \%)$ com idade entre 90 a 99 anos (Figura $2 \mathrm{H}$ ). $\mathrm{O}$ total de óbitos domiciliares em 2020 pelas causas acima mencionadas teve um aumento de $25,88 \%$.

Em contrapartida, houve redução nas mortes hospitalares na ordem de $6,08 \%$ por pneumonia, 3,59\% por IR, 2,21\% por sepse, e um aumento de $338,30 \%$ para SRAG. Essa comparação do total de óbitos hospitalares pelas causas citadas anteriormente mostrou uma redução de 3,24\% em 2020.

Ao se analisar as mortes por causas cardiovasculares em domicílio, notou-se aumento de $18,29 \%$ por AVC (Figura 3A), com maior prevalência em mulheres $(50,32 \%)$ com idade entre 80 e 89 anos (Figura 3B). Também se observou acréscimo de $15,56 \%$ por IAM (Figura 3C), com maior predominância em homens $(61,17 \%)$ entre 60 e 69 anos de idade (Figura 3D). Por fim, identificou-se incremento das mortes em $18,44 \%$ por outras causas cardiovasculares (Figura 3E), com maior predominância em homens (52,47\%) entre 80 e 89 anos de idade (Figura 3F). 0 total de óbitos domiciliares em 2020 pelas causas acima mencionadas comparado ao ano de 2019 teve um aumento de 17,38\%.

Constatou-se decréscimo dos óbitos nas unidades hospitalares de $3,02 \%$ por AVC e um aumento de 3,41\% por IAM e de 2,93\% por outras causas cardiovasculares. 0 total desses óbitos apresentou crescimento de $1,11 \%$. Todavia, ao analisar a partir da SE 12 , nota-se que os óbitos hospitalares são decrescentes em todas as causas cardiovasculares, totalizando uma redução de $3,31 \%$.

\section{DISCUSSÃO}

O presente artigo cumpre seu objetivo primário de avaliar as mortes e seus locais de ocorrência no estado de MG por causas respiratórias e por causas cardiovasculares em 2020. Como citado nos resultados, houve um aumento representativo dos óbitos em domicílio por todas as causas mortis denotadas no objetivo: pneumonia, IR, SRAG, sepse, IAM e AVC, e elas seguem com uma predominância em termo de sexo e faixa etária.

Primeiramente, é de grande importância abordar que a pandemia pela COVID-19 implicou alterações no funcionamento do sistema de saúde, com interrupção de atividades não essenciais ou eletivas que, associadas ao medo de contaminação pelos pacientes, provocaram alterações significativas na dinâmica de atendimento dos serviços hospitalares ${ }^{14}$. Assim, entende-se que essas alterações acarretaram a diminuição do fluxo de pacientes nos hospitais, inclusive daqueles com indicação de atendimento emergencial, ou seja, com risco de vida, e, consequentemente, podem estar colaborando para o aumento de mortes em domicílio.

Em segundo lugar, com relação especificamente à COVID-19, de acordo com o MS, o manejo clínico da síndrome gripal, manifestação clínica relacionada à doença, na Atenção Primária à Saúde (APS) varia de acordo com a gravidade dos casos. Assim, os casos leves devem incluir manejo terapêutico não farmacológico (repouso, hidratação, alimentação adequada, analgésicos e antitérmicos) e isolamento domiciliar por 14 dias com vigilância ativa por profissional da APS, via telefone, a cada $48 \mathrm{~h}^{6}$.

Dessa forma, a proposta de manejo instituída pelo MS prioriza a contenção da disseminação do novo coronavírus (já que o paciente com síndrome gripal leve ficará isolado em domicílio) e a minimização dos gastos, uma vez que o paciente, naquele momento, não possui a necessidade de ocupação de leito hospitalar. Porém, essa medida pode estar contribuindo para o incremento de óbitos em casa. Isso pode ser explicado por não existirem informações completas sobre a história natural da doença, bem como sobre medidas de manejo clínico dos casos de infecção humana pelo SARS-CoV- $2^{6}$, o que as tornam questionáveis. Diante disso, existe certa probabilidade desse paciente evoluir para a forma grave da doença, desenvolvendo a SRAG, e morrer em casa, o que explicaria o aumento das mortes em domicílio por essa causa. Afirma-se isso ao considerar todos os fatores que influenciam essa condição, como as comorbidades, a insipiência da doença, os determinantes sociais e inúmeras outras variáveis que podem impulsionar essa evolução e que envolvem o agente da doença, o paciente e o seu meio ambiente.

A SRAG é um conjunto de sinais e sintomas respiratórios agudos e graves que tem como principais agentes etiológicos os vírus, como influenza, vírus sincicial respiratório (VSR), adenovírus, hantavírus, dengue e coronavírus, e outros agentes, como as bactérias $^{15}$. São considerados casos de SRAG, devendo ser notificados compulsoriamente, os indivíduos com a combinação dos seguintes quadros sintomáticos: febre alta (acima de $37,8^{\circ} \mathrm{C}$ ) $\mathrm{E}$ tosse OU dor de garganta $\mathrm{E}$ dificuldade respiratória OU dispneia OU saturação de $\mathrm{O}_{2}<95 \%$ E necessitou de hospitalização OU veio a óbito tendo apresentado os sintomas referidos, independentemente de hospitalização ${ }^{16}$.

No Brasil, a notificação de casos hospitalizados devido à SRAG passou a ser realizada no Sistema de Informação de Agravos de Notificação (Sinan) desde a pandemia de influenza A (H1N1), em 2009, incluindo o VSR a partir de 2012 e, atualmente, a COVID-19 que, em 2020, tornou a SRAG uma das principais causas de morte no Brasi $^{16}$. Esse cenário influencia diretamente o aumento de mortes domiciliares por SRAG observadas neste estudo no valor de 20,69\% durante o período de pandemia pela COVID-19.

Ainda não é possível fazer uma análise detalhada sobre o perfil epidemiológico de óbitos por SRAG provocados pela COVID-19, porque ainda não existem trabalhos robustos sobre esse tema. Entretanto, segundo Niquini et al. ${ }^{17}$, entre os anos de 2010 e 2019 os agentes infecciosos dominantes foram os vírus Influenza A e B e o VSR. Identificou-se a prevalência em crianças por ambos 


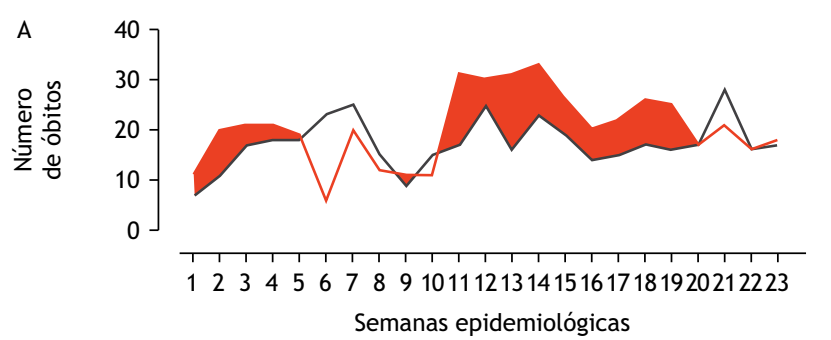

- Mortes por pneumonia em 2019

- Mortes por pneumonia em 2020

- 70 Excesso de mortes por pneumonia em domicílio

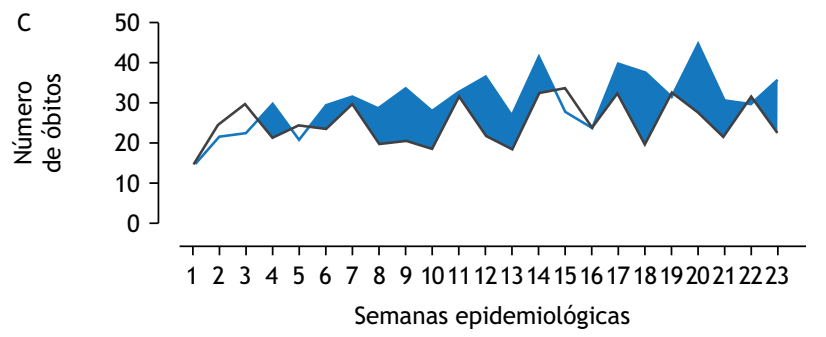

- Mortes por insuficiência respiratória em 2019

- Mortes por insuficiência respiratória em 2020

- 121 Excesso de mortes por insuficiência respiratória em domicílio

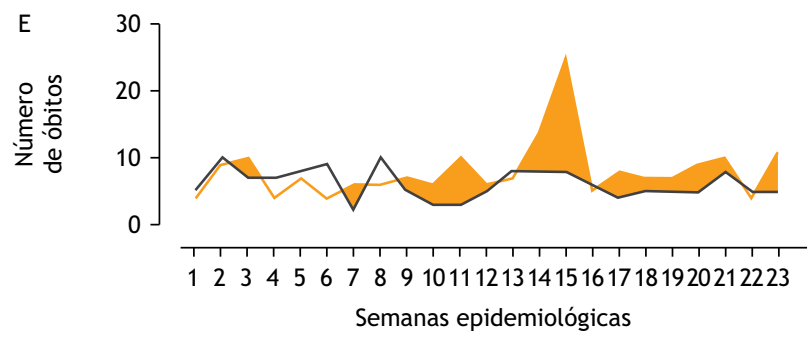

- Mortes por sepse em 2019

- Mortes por sepse em 2020

45 Excesso de mortes por sepse em domicílio

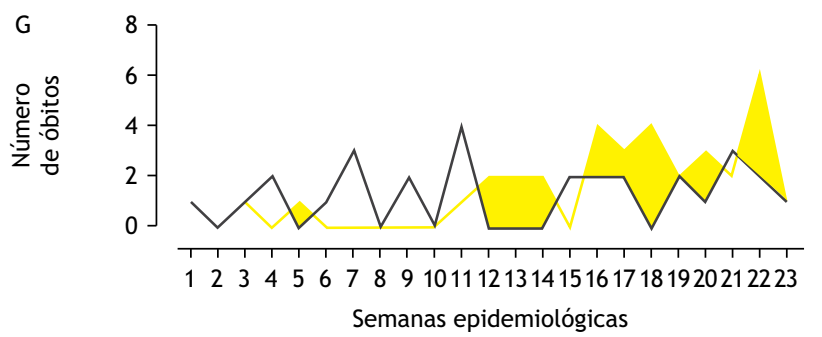

- Mortes por SRAG em 2019

Mortes por SRAG em 2020

6 Excesso de mortes por SRAG em domicílio
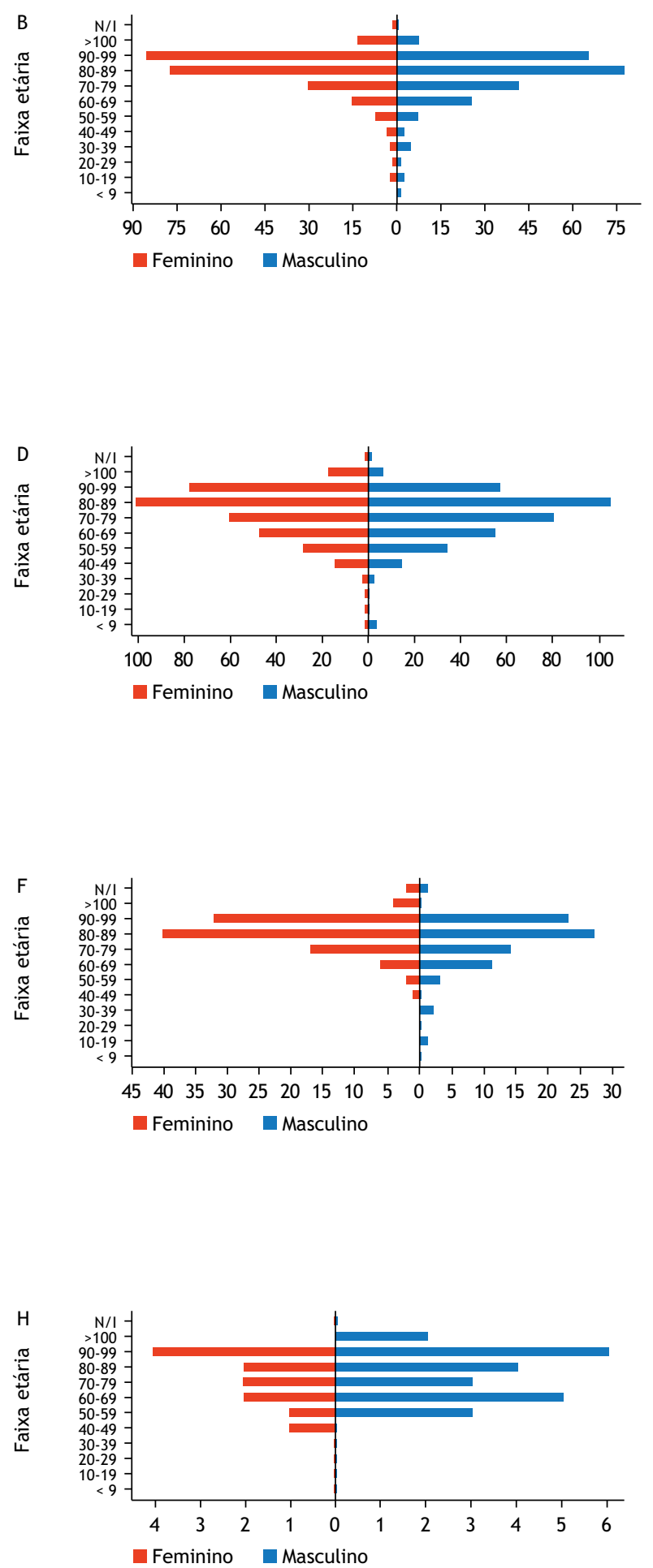

SRAG: Síndrome respiratória aguda grave.

Figura 2. Distribuição de óbitos por causas respiratórias e faixa etária de acordo com o Portal da Transparência do Registro Civil, durante as semanas epidemiológicas de 1 a 23 dos anos 2019 e 2020 no estado de Minas Gerais, Brasil. (A) Excesso de mortes por pneumonia em domicílio; (B) Pirâmide etária de óbitos por pneumonia em 2020; (C) Excesso de mortes por insuficiência respiratória em domicílio; (D) Pirâmide etária de óbitos por

insuficiência respiratória em domicílio em 2020; (E) Excesso de mortes por sepse em domicílio; (F) Pirâmide etária de óbitos por sepse em 2020;

(G) Excesso de mortes por SRAG em domicílio e (H) Pirâmide etária de óbitos por SRAG em 2020. 


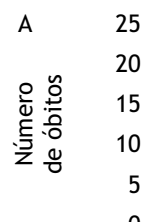

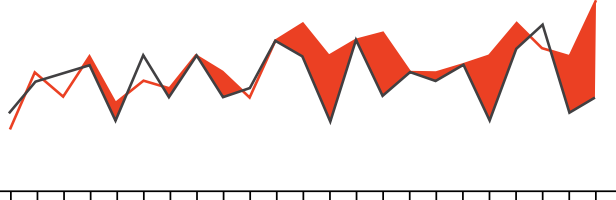

1234567891011121314151617181920212223 Semanas epidemiológicas

- Mortes por AVC em 2019

- Mortes por AVC em 2020

47 Excesso de mortes por AVC em domicílio

C
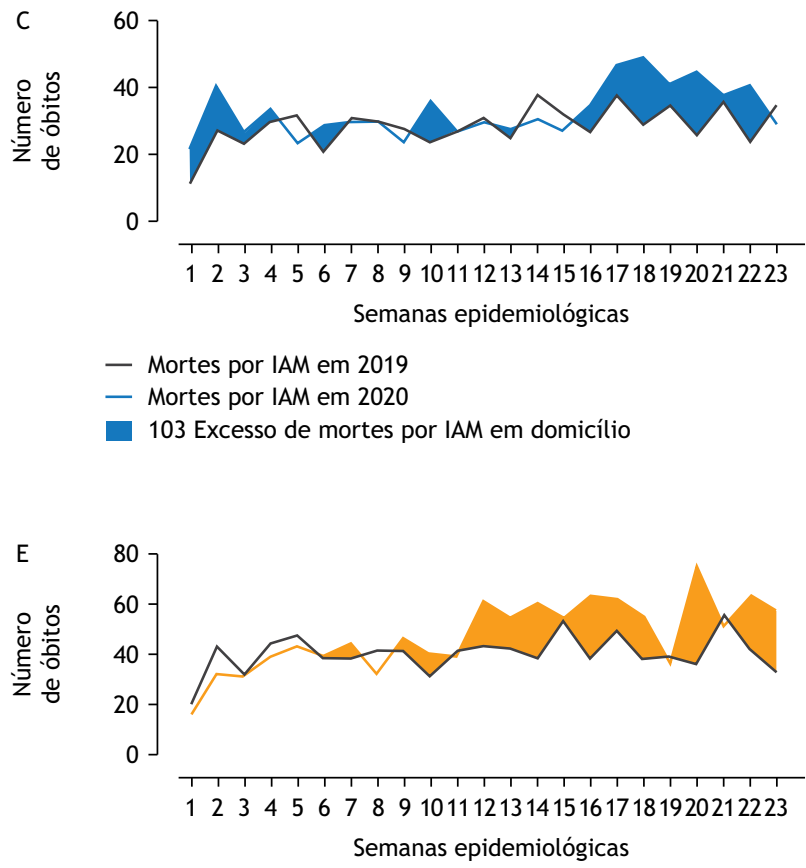

- Mortes por outras causas cardiovasculares em 2019

- Mortes por outras causas cardiovasculares em 2020

170 Excesso de mortes por outras causas cardiovasculares em domicílio

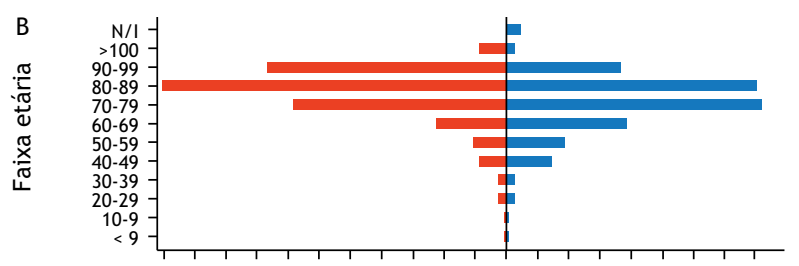

55504540353025201510500510152025303540

Feminino Masculino
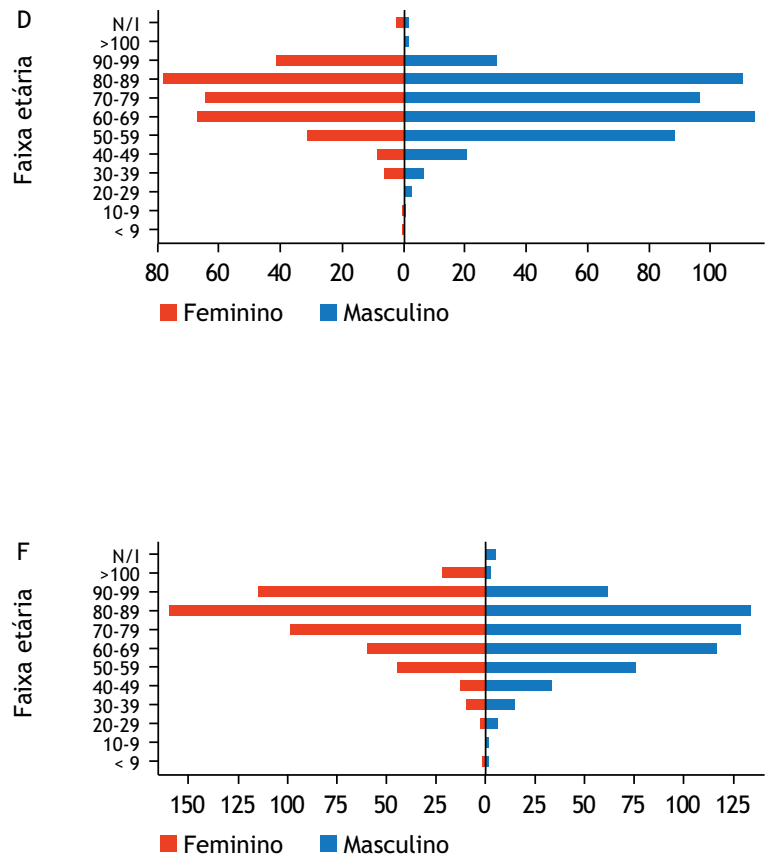

AVC: Acidente vascular cerebral; IAM: Infarto agudo do miocárdio.

Figura 3. Distribuição de óbitos por causas cardiovasculares e faixa etária de acordo com o Portal da Transparência do Registro Civil, durante as semanas epidemiológicas 1 a 23 dos anos de 2019 e 2020 no estado de Minas Gerais, Brasil. (A) Excesso de mortes por AVC em domicílio; (B) Pirâmide etária de óbitos por AVC em 2020; (C) Excessos de mortes por IAM em domicílio; (D) Pirâmide etária de óbitos por IAM em 2020; (E) Excesso de mortes por outras causas cardiovasculares em domicílio; (F) Pirâmide etária de óbitos por outras causas cardiovasculares em 2020.

os vírus, porém, para o vírus de influenza, também há predomínio em idosos com 60 anos ou mais. Por outro lado, as hospitalizações por SRAG em decorrência da COVID-19 concentraram-se entre os homens idosos ou com 40 a 59 anos. Semelhante ao que foi identificado no presente estudo, com predomínio de óbitos domiciliares por SRAG durante a pandemia pela COVID-19 em homens na faixa etária dos 90 a 99 anos e na segunda faixa etária mais acometida dos 60 a 69 anos. Outra questão a se considerar é que o Brasil vive um processo de envelhecimento populacional, com a diminuição da natalidade e da mortalidade, bem como o aumento expressivo de pessoas com mais de 60 anos, caracterizando uma transição demográfica e epidemiológica do país ${ }^{18}$, o que pode contribuir para que essa faixa etária seja uma das mais acometidas.
Nos idosos com multimorbidade já é esperado o curso grave da COVID-19 e, apesar da hospitalização e da terapia intensiva, a mortalidade nesse grupo é alta. Diante dessa realidade, e de esclarecimentos, muitos pacientes optam por permanecer em casa e enfrentar a doença em ambiente familiar ${ }^{19}$. Sabe-se que a maior taxa de mortalidade pela COVID-19 ocorre nesse grupo, principalmente naqueles com comorbidades ${ }^{6}$, e, diante do exposto acima e do receio de infecção pela COVID-19 ao procurar atendimento, é possível a influência dessa conjuntura no aumento do número de óbitos domiciliares.

Em relação aos óbitos por pneumonia em domicílio, os achados da prevalência entre mulheres a partir de 80 anos de idade 
corroboram com um estudo de Dias et al. ${ }^{20}$, o qual demonstra que, entre 2014 e 2019, no Triângulo Mineiro, idosos com 60 anos ou mais foram os principais a serem internados por essa doença. Prina et al. ${ }^{21}$ também mostraram que há uma maior incidência global de pneumonia adquirida na comunidade (PAC) em pessoas acima de 65 anos. Como já mencionado, o distanciamento dos pacientes dos centros de saúde devido ao cenário de pandemia causado pelo novo coronavírus pode ter provocado esse acréscimo. Afirma-se isso, pois, segundo Corrêa et al. ${ }^{22}$, há necessidade de monitoramento constante dos pacientes com PAC, para permitir a avaliação do Índice de Gravidade da Pneumonia (Pneumonia Severity Index, PSI). O PSI é relevante para o manejo adequado do indivíduo, que envolve a decisão sobre o local do atendimento (ambulatorial, hospitalar ou UTI), a necessidade de investigação etiológica e a escolha de antibióticos e sua via de administração. Entre os critérios avaliados no PSI, estão o acompanhamento da pressão arterial, da ureia, da albumina, da frequência respiratória, da taquicardia, da confusão mental, da oxigenação e do pH. Além disso, é recomendado o acompanhamento da saturação de oxigênio $\left(\mathrm{SpO}_{2}\right)$. Assim, fica evidente que o aporte do posto de saúde é essencial para o cuidado desses pacientes, de modo que a manutenção deles em domicílio pode ser fatal.

A ventilação mecânica invasiva via tubo endotraqueal ou cânula traqueal é uma terapia essencial para pacientes com IR aguda ${ }^{23}$. Outras formas de tratamento também envolvem manutenção das vias aéreas e oxigenoterapia ${ }^{24}$. Dessa forma, é imprescindível que esses pacientes tenham acompanhamento hospitalar para que esses serviços sejam fornecidos, o que é limitado no contexto da atual pandemia pelo novo coronavírus.

Além disso, caso o desenvolvimento da IR seja duradouro (por meses ou anos), pode ser desenvolvidas doenças respiratórias crônicas, sendo a doença pulmonar obstrutiva crônica (DPOC) a mais comum ${ }^{24}$. Nesse caso, sabe-se que a atividade física é imprescindível para a reabilitação pulmonar. Treinamento da força do braço ou de vibração do corpo inteiro e, principalmente, o teste prático de caminhada, são essenciais e apresentam resultados benéficos na recuperação desses ${ }^{25}$. Porém, em contexto de pandemia, em que o distanciamento social é preconizado, a realização dessas atividades é dificultada e isso pode agravar os quadros crônicos de IR e resultar em óbito.

Afirma-se isso, pois, segundo Pitanga et al. ${ }^{26}$, é recomendado que, no atual momento, os exercícios sejam realizados preferencialmente com suporte tecnológico, por meio de vídeos com sequências de atividades, aplicativos e orientações online de profissionais. Contudo, como evidenciado pelo presente estudo, as principais vítimas em domicílio por IR são idosos acima de 70 anos, ou seja, é esperado que eles encontrem mais dificuldade de manejar essa situação. Isso pode ocorrer pela inabilidade ou dificuldade em utilizar dispositivos de tecnologia, internet e relacionados ou, simplesmente, por não terem acesso a computador, celular ou tablets. Além disso, também pelo isolamento, essas pessoas estarão sem possibilidade de auxílio de membros da família no uso dessas tecnologias.
As mortes em domicílio por sepse também demonstraram acréscimo em relação ao ano passado e com predominância entre as mulheres no presente estudo, com uma diferença de $9 \%$ entre os sexos. Também foi verificado que a faixa etária entre 80 a 89 anos foi a mais acometida. Ambos os dados corroboram com os achados do estudo de Santos et al. ${ }^{27}$, que avaliou todos os óbitos registrados em 2017 no Sistema de Informação sobre Mortalidade e encontrou predominância de sepse entre mulheres nessa faixa etária.

O diagnóstico dessa patologia é o obstáculo inicial a ser enfrentado pelos médicos, pois suas primeiras manifestações podem ser confundidas com outros processos não infecciosos ${ }^{28}$. Essas constatações podem auxiliar no entendimento do aumento das mortes em domicílio em 2020 por essa doença em MG, pois, se seu reconhecimento já é difícil presencialmente pelo médico e mediante exames laboratoriais, isso pode ser ainda mais negligenciado em atendimentos remotos ou pela não procura por serviços hospitalares.

Carvalho e Trotta ${ }^{28}$ também destacaram que a sepse pode resultar em disfunção, falência de um ou mais órgãos ou em óbito. Assim, o Conselho Federal de Medicina preconiza que pacientes com essa reação devem ser transferidos o mais rápido possível para as unidades de cuidados intermediários ou para as UTI a fim de prosseguirem com o tratamento. Nesse âmbito, o contexto da diminuição dos atendimentos ambulatoriais e da ida ao hospital devido ao cenário provocado pela COVID-19 pode propiciar óbitos em domicílio por essa causa, visto que a intervenção médica se mostra crucial para conter essa condição.

O IAM é a principal causa de morte no Brasil e no mundo ${ }^{29}$. Em diversas regiões do globo, notou-se queda importante de procura por atendimento por IAM devido à pandemia pela COVID-19, que podem variar de $50 \%$ a $90 \%$ de redução ${ }^{30}$. Sabe-se que a maioria das mortes por IAM ocorre nas primeiras horas de manifestação da doença: $40 \%$ a $65 \%$ na primeira hora e aproximadamente $80 \%$ nas primeiras $24 \mathrm{~h}$. Assim, a maior parte das mortes acontece fora do ambiente hospitalar e, geralmente, é desassistida pelos médicos. Por isso, são imprescindíveis o atendimento precoce e a rápida instituição de tratamento adequado na sobrevida do paciente com IAM ${ }^{31}$. Nessa perspectiva, a quarentena, por priorizar o isolamento social e a consequente permanência em casa, pode ampliar ainda mais a probabilidade de um paciente com IAM vir a óbito em domicílio. Neste estudo, observou-se incremento de $15,56 \%$ nas mortes domiciliares por essa afecção durante a pandemia pela COVID-19, o que corrobora com essa suspeição.

Vale considerar que, no Brasil, os cartórios registraram um aumento de $31 \%$ no número de mortes por doenças cardiovasculares ${ }^{12}$ que, em parte, poderia ser um reflexo desse não atendimento ao paciente com IAM e o seu consequente óbito. Entretanto, é possível que esse aumento também esteja sendo influenciado pela subnotificação de óbitos pela COVID-19, porque, principalmente devido às dificuldades de diagnóstico e os riscos de contaminação, muitas declarações de óbito podem estar sendo expedidas com a causa mortis por parada cardiorrespiratória, se enquadrando em doença cardiovascular, e não por COVID-19. 
Com relação ao perfil epidemiológico, nesse estudo encontrou-se maior incidência de óbitos em domicílio por IAM em homens com idade entre 60 e 69 anos, assim como encontrado por Marino et al. ${ }^{29}$ para perfil de pacientes atendidos por IAM em estudo realizado na região Norte de $M G$, no qual $67,6 \%$ eram do sexo masculino e a média de idade era de 63 anos.

O AVC é a segunda principal causa de morte no mundo e no Brasil. É uma das mais importantes doenças crônicas não transmissíveis que resultam em mortes e em internações, além de causar algum tipo de deficiência parcial ou completa ${ }^{32}$. Ele pode ser do tipo isquêmico, correspondendo a $80 \%$ dos casos, ou do tipo hemorrágico em $20 \%$ dos $\operatorname{casos}^{33}$. É uma emergência neurológica e a perda de tempo para a abordagem dos pacientes significa uma pior evolução. Por isso, é necessário identificar rapidamente os sinais e sintomas do AVC, procurar atendimento médico hospitalar especializado, ou seja, uma unidade de AVC, realizar os exames diagnósticos e o tratamento específico em tempo hábil, seja ele medicamentoso, clínico ou cirúrgico, para evitar mortalidade e sequelas ${ }^{34}$. Dessa forma, assim como o exposto para o IAM, devido à necessidade de atendimento imediato ou por medo de contaminação pela COVID-19, os sinais e sintomas do AVC podem estar sendo negligenciados pelos pacientes. Isso pode explicar o acréscimo de 18,29\% nas mortes em domicílio por AVC observadas neste estudo.

Sobre o perfil epidemiológico dessa doença, Mourão et al..$^{35}$ encontraram, em um hospital credenciado na linha de cuidados de AVC em MG, a prevalência no sexo masculino e a idade média de 64,3 anos para os pacientes atendimentos por AVC. Com relação ao perfil de óbitos por AVC, no estado do Paraná, Hata et al. ${ }^{33}$ verificaram que mais da metade eram pacientes masculinos com maior incidência na faixa etária dos 70 aos 79 anos. Já nos achados deste artigo, o perfil de óbitos por AVC em 2020, no período analisado apresenta prevalência entre mulheres com idade entre 80 e 89 anos.

\section{CONCLUSÕES}

O presente artigo traz como contribuição o destaque para o crescente número de mortes em domicílio por causas respiratórias semelhantes às da COVID-19 e por causas cardiovasculares e suas correlações entre a faixa etária e o sexo mais acometidos. Esses dados podem contribuir com a criação de políticas públicas que abordem o correto manejo dessas condições em concomitância ao controle do novo coronavírus.

As condições de cuidado impostas pela atual pandemia preconizam distanciamento social e redução dos serviços de saúde oferecidos. Ambas resoluções diminuem a procura por atendimento por parte da população e são medidas essenciais para a contenção do avanço da COVID-19. Porém, diante dos dados aqui expostos, nota-se a necessidade de se ter um olhar mais apurado para doenças e para manifestações que requerem acompanhamento médico contínuo e suporte tecnológico presente nos hospitais, como nos casos das patologias aqui apresentadas. Nesse sentido, é imperioso que estudos similares a este sejam feitos em outras regiões, para averiguar se esse padrão se repete, de modo a certificar a influência nacional do novo coronavírus sobre as mortes em domicílio, tanto pelas causas aqui analisadas, como por outras.

Este artigo tem como limitação a oferta de dados secundários, a qual se restringe aos anos de 2019 e 2020, o que impossibilita uma comparação em relação aos outros anos. Além disso, por serem dados de um sistema governamental, pode haver atrasos em suas atualizações, o que prejudica a precisão dos valores. Por fim, não foi possível averiguar se houve diminuição da procura por atendimento hospitalar, ao comparar a entrada de pacientes nos serviços de saúde de 2020 com 2019, pois esses resultados não estão disponíveis. Desse modo, a diminuição das mortes em hospitais pelas causas aqui analisadas é em relação ao valor bruto desses óbitos, e não com base na relação entre entrada no hospital e morte nesse ambiente.

Após analisar cuidadosamente tanto as bases de dados disponíveis como a literatura afins, pudemos explorar os aspectos relacionados entre essa nova doença e sua implicação na política de tratamentos em domicílio, o que traz um novo olhar para a situação atual.

\section{REFERÊNCIAS}

1. Shi Y, Wang G, Cai XP, Deng JW, Zheng L, Zhu $\mathrm{HH}$ et al. An overview of COVID-19. J Zhejiang Univ Sci B. 2020;21(5):343-60. https://doi.org/10.1631/jzus.B2000083

2. Johns Hopkins University and Medicine - JHU. COVID-19 dashboard by the center for systems science and engineering (CSSE). Baltimore: Johns Hopkins University and Medicine; 2020[acesso 17 jul 2020]. Disponível em: https://coronavirus.jhu.edu/map.html

3. Secretaria de Estado de Saúde de Minas Gerais - SES-MG. Boletim epidemiológico e assistencial COVID-19: edição especial número 8. Belo Horizonte: Secretaria de Estado de Saúde de Minas Gerais; 2020[acesso 17 jul 2020]. Disponível em: https://www.saude.mg.gov.br/images/
noticias_e_eventos/000_2020/jun-jul-ago/17-06-Boletim_ Edicao-EspecialN8.pdf

4. Fundação Oswaldo Cruz - Fiocruz. Risco de espalhamento da COVID-19 e avaliação da vulnerabilidade socioeconômica por estado: Minas Gerais. Boletim Minas Gerais. 2 abr 2020[acesso 17 jul 2020]. Disponível em: https:// gitlab.procc. fiocruz.br/mave/repo/-/blob/master/ Relat\%C3\%B3rios\%20t\%C3\%A9cnicos\%20-\%20COVID-19/ relat $\%$ C3\%B3rio\%20t\%C3\%A9cnico\%203\%20-\%20boletins $\% 20$ estaduais-2020-04-01/boletim_estadualMG.pdf

5. Alves THE, Souza TA, Silva SA, Ramos NA, Oliveira SV. Underreporting of death by COVID-19 in Brazil's second most populous state. MedRxiv. 2020:1-15. https://doi.org/10.1101/2020.05.20.20108415 
6. Ministério da Saúde (BR). Protocolo de manejo clínico do coronavírus (COVID-19) na atenção primária à saúde. Brasília: Ministério da Saúde; 2020[acesso 17 jul 2020]. Disponível em: https://www.saude.gov.br/images/ pdf/2020/marco/20/20200318-ProtocoloManejo-ver002.pdf

7. Secretaria de Estado de Saúde de Minas Gerais - SES-MG. Saúde digital em Minas Gerais. Belo Horizonte: Secretaria de Estado de Saúde de Minas Gerais; 2020[acesso 16 jul 2020]. Disponível em: http://coronavirus.saude.mg.gov.br/saudedigitalmg

8. Agência Nacional de Saúde Suplementar - ANS. ANS orienta: consultas, exames e cirurgias que não sejam urgentes devem ser adiados. Rio de Janeiro: Agência Nacional de Saúde Suplementar; 2020[acesso 17 jul 2020]. Disponível em: http://www.ans.gov.br/aans/noticias-ans/ consumidor/5426-ans-orienta-consultas-exames-e-cirurgiasque-nao-sejam-urgentes-devem-ser-adiados

9. Instituto Brasileiro de Geografia e Estatística - IBGE. Panorama: Minas Gerais. Brasília: Instituto Brasileiro de Geografia e Estatística; 2020[acesso 17 abr 2020]. Disponível em: https://cidades.ibge.gov.br/brasil/mg/panorama

10. Atlas Brasil. Atlas do desenvolvimento no Brasil: Minas Gerais. Brasília: Instituto de Pesquisa Econômica Aplicada; 2020[acesso 29 abr 2020]. Disponível em: http://www.atlasbrasil.org.br/2013/pt/perfil_uf/ minas-gerais\#demografia

11. Associação dos Registradores de Pessoas Naturais ARPEN Brasil. Registros. Portal de Serviços Registro Civil. 2020[acesso 29 abr 2020]. Disponível em: https://transparencia.registrocivil.org.br/registros

12. Associação dos Registradores de Pessoas Naturais ARPEN Brasil. Painel registral: óbitos com suspeita ou confirmação de COVID-19. Portal de Serviços Registro Civil: especial COVID. 2020[acesso 29 abr 2020]. Disponível em: https: / / transparencia.registrocivil.org.br/especial-covid

13. Associação dos Registradores de Pessoas Naturais ARPEN Brasil. Painel registral: óbitos com suspeita ou confirmação de COVID-19. Portal de Serviços Registro Civil: Registral COVID. 2020[acesso 29 abr 2020]. Disponível em: https: / / transparencia.registrocivil.org.br/registral-covid

14. Oliveira M, Sousa P, Sousa L, Mendes C, Labucha M, Macedo F. A cardiologia na pandemia COVID-19: que impacto na atividade clínica? Gaz Med. 2020;2(7):101-6. https://doi.org/10.29315/gm.v7i2.348

15. Ribeiro SA, Brasileiro GS, Soleiman LNC, Silva CC, Kavaguti CS. Síndrome respiratória aguda grave causada por influenza A (subtipo H1N1). J Bras Pneumol. 2020;36(3):386-9. https://doi.org/10.1590/S1806-37132010000300017

16. Bastos LS, Niquini RP, Lana RM, Villela DAM, Crus OG, Coelho FC et al. COVID-19 e hospitalizações por SRAG no Brasil: uma comparação até a $12^{\text {a }}$ semana epidemiológica de 2020. Cad Saude Publica. 2020;36(4):1-8. https://doi.org/10.1590/0102-311×00070120

17. Niquini RP, Lana RM, Pacheco AG, Cruz OG, Coelho FC, Carvalho LM et al. SRAG por COVID-19 no Brasil: descrição e comparação de características demográficas e comorbidades com SRAG por influenza e com a população geral. Cad Saude Publica. 2020;36(7):1-12. https://doi.org/10.1590/0102-311X00149420

18. Oliveira AS. Transição demográfica, transição epidemiológica e envelhecimento populacional no Brasil. Hygeia. 2019;15(31):69-79. https://doi.org/10.14393/Hygeia153248614

19. Kunz R, Minder M. COVID-19 pandemic: palliative care for elderly and frail patients at home and in residential and nursing homes. Swiss Med Wkly. 2020;150:1-2. https://doi.org/10.4414/smw.2020.20235

20. Dias FLT, Mendonça FD, Pinto GM, Borges ISC, Oliveira SV. Doenças respiratórias no triângulo mineiro: análise epidemiológica e projetiva com a pandemia de COVID-19. J Health Biol Sci. 2020;8(1):1-6. https://doi.org/10.12662/2317-3219jhbs.v8i1.3206.p1-6.2020

21. Prina E, Ranzani OT, Torres A. Community-acquired pneumonia. Lancet. 2015;386(9998):1097-108. https://doi.org/10.1016/S0140-6736(15)60733-4

22. Corrêa RA, Costa AN, Lundgren F, Michelin L, Figueiredo MR, Holanda $M$ et al. 2018 recommendations for the management of community acquired pneumonia. J Bras Pneumol. 2018;44(5):405-23. https://doi.org/10.1590/S1806-37562018000000130

23. Fichtner F, Moerer O, Weber-Carstens S, Nothacker M, Kaisers $\mathrm{U}$, Laudi $\mathrm{S}$. Clinical guideline for treating acute respiratory insufficiency with invasive ventilation and extracorporeal membrane oxygenation: evidence-based recommendations for choosing modes and setting parameters of mechanical ventilation. Respiration. 2019;98(4):357-72. https://doi.org/10.1159/000502157

24. Pádua Al, Alvares F, Martinez JAB. Insuficiência respiratória. Medicina. 2003;36(2/4):205-13. https://doi.org/10.11606/issn.2176-7262.v36i2/4p205-213

25. Spruit MA, Rochester CL, Pitta F, Kenn K, Schols AMWJ, Hart $\mathrm{N}$ et al. Pulmonary rehabilitation, physical activity, respiratory failure and palliative respiratory care. Thorax. 2018;74(7):693-9. https://doi.org/10.1136/thoraxjnl-2018-212044

26. Pitanga FJG, Beck CC, Pitanga CPS. Atividade física e redução do comportamento sedentário durante a pandemia do coronavírus. Arq Bras Cardiol. 2020:1-3. https://doi.org/10.36660/abc.2020023

27. Santos MR, Cunha CC, Ishitani LH, França EB. Mortes por sepse: causas básicas do óbito após investigação em 60 municípios do Brasil em 2017. Rev Bras Epidemiol. 2020;22(Supl 3):1-13. https://doi.org/10.1590/1980-549720190012.supl.3

28. Carvalho PRA, Trotta EA. Avanços no diagnóstico e tratamento da sepse. J Pediatr. 2003;79(Supl 2):S195-S204. https: / /doi.org/10.1590/\$0021-75572003000800009

29. Marino BCA, Marcolino MS, Reis Júnior RDS, França ALN, Passos PFDO, Lemos TR et al. Perfil epidemiológico e indicadores de qualidade em pacientes com síndrome coronariana aguda na região norte de Minas Gerais: projeto 
Minas telecardio 2. Arq Bras Cardiol. 2016;107(2):106-15. https://doi.org/10.5935/abc.20160095

30. Wood S. The mystery of the missing STEMIs during the COVID-19 pandemic. Tctmd Feature: Interventional. 2 abr 2020[acesso 17 jul 2020]. Disponível em: https://www.tctmd.com/news/ mystery-missing-stemis-during-COVID-19-pandemic

31. Piegas LS, Timerman A, Feitosa GS, Nicolau JC, Mattos LAP, Andrade MD et al. $V$ diretriz da sociedade brasileira de cardiologia sobre tratamento do infarto agudo do miocárdio com supradesnível do segmento ST. Arq Bras Cardiol. 2015;105(2 supl 1):1-105. https://doi.org/10.5935/abc.20150057

32. Almeida SRM. Análise epidemiológica do acidente vascular cerebral no Brasil. Rev Neurocienc. 2012;20(4):481-2. https://doi.org/10.4181/RNC.2012.20.483ed.2p
33. Hata MM, Rodrigues AJS, Quadros AC, Turmina L, lachinski RE, Osório APS. Análise do perfil epidemiológico de óbitos por doenças cerebrovasculares em residentes do estado do Paraná no período de 2008 a 2017. Fag J Health. 2019;1(3):209-15. https://doi.org/10.35984/fjh.v1i3.126

34. Gagliardi RJ, Raffin CN, Fábio SRC. Tratamento da fase aguda do acidente vascular cerebral. São Paulo: Academia Brasileira de Neurologia; 2001[acesso 18 jul 2020]. Disponível em: https://diretrizes.amb.org.br/_BibliotecaAntiga/tratamentoda-fase-aguda-do-acidente-vascular-cerebral.pdf

35. Mourão AM, Vicente LCC, Chaves TS, Santanna RV, Meira FDC, Xavier RMDB et al. Perfil dos pacientes com diagnóstico de AVC atendidos em um hospital de Minas Gerais credenciado na linha de cuidados. Rev Bras Neuro. 2017;53(4):12-6.

Contribuição dos Autores

Alves THE, Souza TA, Silva SA, Ramos NA, Oliveira SV - Concepção, planejamento (desenho do estudo), aquisição, análise, interpretação dos dados e redação do trabalho. Os autores aprovaram a versão final do trabalho.

Conflito de Interesse

Os autores informam não haver qualquer potencial conflito de interesse com pares e instituições, políticos ou financeiros deste estudo.

Esta publicação está sob a licença Creative Commons Atribuição 3.0 não Adaptada.

Para ver uma cópia desta licença, visite http://creativecommons.org/licenses/by/3.0/deed.pt_BR. 